$\mathbf{R}_{\text {ESEARCH }} \mathbf{P}_{\text {APER }} \longrightarrow \frac{\text { FOOD SCIENCE }}{\text { RESEARCH JOURNAL }}$

\title{
Development of omega-3 rich food products using soybean and flaxseed
}

\author{
Mamta Jaiswal and Shalini Agrahari
}

\begin{abstract}
The objective of present investigation "Development of omega-3 rich food products using soybean and flaxseed" was to standardize and develop the products using soybean and flaxseed and their organoleptic evaluation. Omega-3 polyunsaturated fatty acids play a crucial role in reduction of cardiovascular disease risk factors. Dietary source of omega-3 polyunsaturated fatty acid as eicosapentaenoic acid (EPA) and docosahexaenic acid (DHA) which include fatty fish (salmon and herring), linolenic acid can be obtained from vegetable oils (canola oil, soybean oil and olive oil), walnuts and pistachio. Omega -3 fatty acids improve endothelial function, reduce inflammatory process and ischemic heart disease. Regulation of influx and efflux of sodium and calcium ions through omega- 3 fatty acids helps to prevent cardiac arrhythmia. Fish oil may enhance cardiac remodeling in heart failure. Linolenic acid is recommended for an individual without cardiovascular disease whereas at least $1 \mathrm{~g}$ per day of omega-3 polyunsaturated fatty acids should be consumed by patient with cardiovascular disease. Developed products of soybean and flaxseed were soybean halwa and soybean and flaxseed pancake. The organoleptic evaluation of products was done by using (9-Point Hedonic Scale). The result of soybean and flaxseed based 2 products for soybean halwa, soybean and flaxseed pancake $\left(\mathrm{T}_{0}\right)$ and $\left(\mathrm{T}_{1}\right)$ were best in all treatments in case of all sensory attributes.
\end{abstract}

Key Words : Omega-3, Polyunsaturated fatty acids, Cardiovascular disease, Eicosanoids, Soybean, Flaxseed

How to cite this article : Jaiswal, Mamta and Agrahari, Shalini (2018). Development of omega-3 rich food products using soybean and flaxseed. Food Sci. Res. J., 9(1): 102-105, DOI : 10.15740/HAS/FSRJ/9.1/102-105. 